\title{
Kearns-Sayre syndrome - rare cause of growth hormone deficiency
}

Authors: Dora Bulić ${ }^{1}$, Duje Braovac ${ }^{2}$, Maja Vinković ${ }^{2}$, Katja Dumić Kubat ${ }^{1,2}$

${ }^{1}$ University of Zagreb, School of Medicine, Zagreb, Croatia

${ }^{2}$ Department of Pediatrics, Division for Endocrinology and Diabetes, University Hospital Centre Zagreb, Zagreb, Croatia

DOI: https://doi.org/10.26800/LV-142-supp5-18

\section{Introduction:}

Kearns - Sayre syndrome (KSS) is a rare mitochondriopathy. Among chronic progressive external ophthalmoplegia, pigmentary retinal degeneration and ataxia that are present in most patients before age of 20 , majority of patients also have various endocrine deficiencies.

\section{Case report:}

We report on a previously healthy 8-year-old boy who presented to the endocrine clinic due to short stature (height $116 \mathrm{~cm} ;-2.15 \mathrm{SD}$, weight $18 \mathrm{~kg} ;-2.2 \mathrm{SD}$ ). He had presented with mild unilateral ptosis and was a bit clumsy, while the rest of clinical status was unremarkable. Growth hormone $(\mathrm{GH})$ stimulation tests were performed (L-DOPA and insulin tolerance test), showing growth hormone deficiency. Before $\mathrm{GH}$ treatment introduction brain MRI was performed showing hyperintensities in the globus. Additional workup was done- revealing pigmented retinitis, external ophthalmoplegia, high levels of lactate and protein in cerebrospinal fluid. Diagnosis of KSS was established and molecularly confirmed in urine samples (large $7.6 \mathrm{~kb}$ deletion described in 65\% mtDNA). After diagnosis confirmation, parents initially decided not to introduce GH treatment. After 2 years, neurological symptoms including ophthalmoplegia and ataxia progressed and the patient grew only $4 \mathrm{~cm}$. Subsequently, GH treatment was introduced with good effect on growth and no side effects. At the age of 12 , impaired glucose tolerance was observed.

\section{Conclusion:}

KSS is a rare and slow progressive disorder. Despite initial symptoms starting at early age, diagnosis is usually much later confirmed. Heart block is the most severe complication of disease. Short stature and GH deficiency can be a part of KSS and are usually evident before onset of visible neurological symptoms. Early diagnosis is very important in order to introduce endocrine treatment, routinely screen for comorbidities that are part of KSS, improve quality of life and prevent complications and sudden death.

Keywords: endocrinopathy, growth hormone deficiency, Kearns-Sayre syndrome, ophthalmoplegia 